\title{
Molecular Characterization of Cryptosporidium spp. in Wild Rodents of Southwestern Iran Using 18s rRNA Gene Nested-PCR-RFLP and Sequencing Techniques
}

\author{
Jasem Saki, ${ }^{1,2}$ Masoud Foroutan-Rad, ${ }^{3}$ and Reza Asadpouri ${ }^{2}$ \\ ${ }^{1}$ Health Research Institute, Infectious and Tropical Diseases Research Center, Ahvaz Jundishapur University of Medical Science, \\ Ahvaz, Iran \\ ${ }^{2}$ Department of Medical Parasitology, Faculty of Medicine, Ahvaz Jundishapur University of Medical Sciences, Ahvaz, Iran \\ ${ }^{3}$ Department of Parasitology, Faculty of Medical Sciences, Tarbiat Modares University, Tehran, Iran
}

Correspondence should be addressed to Masoud Foroutan-Rad; masoud_foroutan_rad@yahoo.com

Received 1 August 2016; Revised 5 October 2016; Accepted 26 October 2016

Academic Editor: Jean-Paul J. Gonzalez

Copyright (C) 2016 Jasem Saki et al. This is an open access article distributed under the Creative Commons Attribution License, which permits unrestricted use, distribution, and reproduction in any medium, provided the original work is properly cited.

\begin{abstract}
Background. Rodents could act as reservoir for Cryptosporidium spp. specially C. parvum, a zoonotic agent responsible for human infections. Since there is no information about Cryptosporidium infection in rodents of Ahvaz city, southwest of Iran, hence, this survey was performed to determine the prevalence and molecular characterization of Cryptosporidium spp. in this region. Materials and Methods. One hundred rodents were trapped from different regions of Ahvaz city. Intestine contents and fecal specimens of rodents were studied using both microscopy examination to identify oocyst and nested-polymerase chain reaction (PCR) technique for 18s rRNA gene detection. Eventually restriction fragment length polymorphism (RFLP) method using $S s p I$ and $V s p I$ restriction enzymes was carried out to genotype the species and then obtained results were sequenced. Results. Three out of 100 samples were diagnosed as positive and overall prevalence of Cryptosporidium spp. was 3\% using both modified Ziehl-Neelsen staining under light microscope and nested-PCR $(830 \mathrm{bp})$ methods. Afterwards, PCR-RFLP was performed on positive samples and C. parvum pattern was identified. Finally PCR-RFLP findings were sequenced and presence of C. parvum was confirmed again. Conclusions. Our study showed rodents could be potential reservoir for C. parvum. So an integrated program for control and combat with them should be adopted and continued.
\end{abstract}

\section{Introduction}

Cryptosporidium spp. are ubiquitous intracellular protozoan parasites that affect wide range of vertebrates like human and livestock [1]. Oocysts are infective stage of life cycle released via feces from infected hosts to environment in large amount. They are resistant to various environmental alterations and remain infective for long period in appropriate condition like surface of water and moist soils; thus, they are liable for transmission of disease to animals and humans $[2,3]$. Cryptosporidium as an opportunistic agent infects the alimentary tract of hosts and have wide spectrum of clinical symptoms in human ranging from self-limiting and asymptomatic in immunocompetent individuals to severe and life-threatening in immunocompromised persons $[2,4,5]$. For first time, human cryptosporidiosis was diagnosed in individuals who had severe watery diarrhea during 1970s [6].

During past decades, numerous evidences have confirmed that small mammals such as rodents are considered as carrier or reservoir for several infectious agents. They can transmit the pathogens directly or indirectly. Also role of rodents in transferring the helminth and protozoan infections are clear [7-10]. Surveys in different zones of Iran indicate the existence of various rodent species such as the house mice (Mus musculus), the black rat (Rattus rattus), the brown rat (Rattus norvegicus), and the Himalayan rat (Rattus pectoris) in country $[8,10,11]$. Based on our previous survey in Ahvaz district, southwest of Iran, rodents could be considered as potential source of Toxoplasma gondii infection for definitive hosts [9]. 
Recently, several molecular studies have been conducted to determine the Cryptosporidium genotypes. Small subunitrRNA (SSU 18s rRNA) gene is being utilized for identification of Cryptosporidium spp. worldwide extensively. According to SSU-rRNA gene sequencing, at least 20 Cryptosporidium species have been identified and more than sixty Cryptosporidium genotypes have undeterminate status till now. Approximately eight Cryptosporidium species/genotypes including C. parvum, C. hominis, C. felis, C. meleagridis, C. ubiquitum, C. viatorum, C. canis, and C. cuniculus are the main species responsible for human infections, although C. hominis and C. parvum account for over $90 \%$ of human cryptosporidiosis worldwide $[1,2]$.

Numerous epidemiological surveys have been performed throughout the globe and prevalence of Cryptosporidium infection in rodents was highly varied from $63 \%$ in UK [12], 32.8\% in United States of America (USA) [13], 24.3\% in Italy [14], $7.6 \%$ in Maryland [15], $11.5 \%$ in China [16], $25.8 \%$ in Philippines [17], 8.2\% in northern Australia [18], $0 \%$ in northeast of Iran (Mashhad city) [19], and $27.3 \%$ in north of Iran (Tehran city) [20]. Due to lack of reports about Cryptosporidium infection in rodents of southwest of Iran till now, current study was aimed to determine the prevalence and molecular characterization of Cryptosporidium spp. in this region.

\section{Materials and Methods}

2.1. Study Area. Ahvaz city, capital of Khuzestan province which is located in the southwest of Iran $\left(31^{\circ} 50^{\prime} \mathrm{N}\right.$ and $49^{\circ}$ $11^{\prime} \mathrm{E}$ ), is ranked as the 7th largest city throughout the country and based on the latest census, its population was calculated at 1,395,184 in 352,128 families. Weather temperature is highly variable throughout the year so that in summer temperature exceeds $50^{\circ} \mathrm{C}$ whereas in winter it falls to $5^{\circ} \mathrm{C}$. Also, annual average rainfall is approximately $230 \mathrm{~mm}$. There is high density of rodents species and rat-man-domestic animals adjacency is remarkable in Ahvaz city $[9,11]$. Rodents are additional reservoir for Cryptosporidium spp., mostly preyed on by cats and dogs, and, hence, could spread parasitic infections via other animals [8].

2.2. Rodents Collection. Ahvaz city initially divided into five geographical locations (north, west, south, east, and center). In each location Sherman live traps were placed outdoor at the entrance of rodent colonies and baited with favorite piece of foods (including cucumber pieces, tomato, and roasted almonds). The Sherman live traps were installed at sunset and gathered before sunrise. Overall, 100 rodents were collected from three different species (6 M. musculus, 73 R. norvegicus, and $21 R$. rattus). Eventually trapped rodents were gathered and transferred to Department of Medical Parasitology of Ahvaz JundishapurUniversity of Medical Sciences. The trapped rodents were anaesthetized by putting the live traps in a thick transparent polythene bag and then a cotton swab was soaked in ether and placed near their nose. Afterwards, the anaesthetized rodents were dissected and fecal samples gathered from rectum or large intestinal section. Skull and tooth structures were used for species identification. The Iranian rodent key of Etemad was performed to identify the rodents [21].

2.3. Detection of Cryptosporidium spp. Oocysts. Samples were collected from intestine contents and fecal specimens of rodents. After sugar flotation (SG 1.266; $128 \mathrm{~g}$ sucrose and 100 distilled water) [22] and modified Ziehl-Neelsen staining, the samples were examined to find the Cryptosporidium spp. oocysts using optical microscope under $\times 1000$ magnification. Diagnosis of oocysts was based on morphological features like red spherical shapes. Finally the samples were maintained at $2.5 \%$ potassium dichromate $\left(\mathrm{K}_{2} \mathrm{Cr}_{2} \mathrm{O}_{7}\right)$ [23] and kept in refrigerator (1-2 weeks) until DNA was extracted.

2.4. DNA Extraction and Nested-PCR. DNA extraction procedure was performed using QIAamp ${ }^{\circledR}$ DNA stool mini kit (QIAamp DNA Stool Mini Kit, USA) based on the manufacturer's guideline. The extracted DNA was kept in $-20^{\circ} \mathrm{C}$ for next tests. For nested-PCR method, we used two specific primers to detect 18s rRNA gene whose length of produced fragments was $1325 \mathrm{bp}$ and $830 \mathrm{bp}$. This dual stages technique was run using different primers that primary and secondary stages primers were as following: $[4,20,23,24]$.

\section{First Stage Primers}

Forward (F1): $5^{\prime}$-TTCTAGAGCTAATACATGCG-3'
Reverse (R1): $5^{\prime}$-CCCATTTCCTTCGAAACAGGA-
$3^{\prime}$

Second Stage Primers

\section{Forward (F2): 5'-GGAAGGGTTGTATTTATT - AGATAAAG-3' \\ Reverse (R2): 5'-CTCATAAGGTGCTGAAGG- AGTA-3'}

Finally PCR products after loading on $1.5 \%$ agarose gel and electrophoresis for 1.5 hours were stained with ethidium bromide and then visualized under UV light using Gel Doc device (Uvidoc, Gel Documentation System, Cambridge, UK) [25].

2.5. Genotyping Cryptosporidium spp. Using 18s rRNA PCRRFLP and Sequencing. RFLP assay was done to determine the Cryptosporidium spp., using digestion of secondary PCR products. For this purpose, SspI and VspI endonuclease enzymes were employed for species recognition and genotyping, respectively, based on manufacturer's protocol as earlier described [20]. Then the mixture was incubated for approximately 8 hours at $37^{\circ} \mathrm{C}$. Eventually visualization of the digested products was carried out under UV transilluminator after $1.5 \%$ agarose gel electrophoresis and ethidium bromide staining $[20,26]$. The nested-PCR positive samples were purified using Bioneer kit corporation (Korea) and were sequenced by the same corporation. Sequence alignments were constructed by CLUSTAL W software (http://www.ddbj.nig.ac.jp/search/clustalwe.html). 


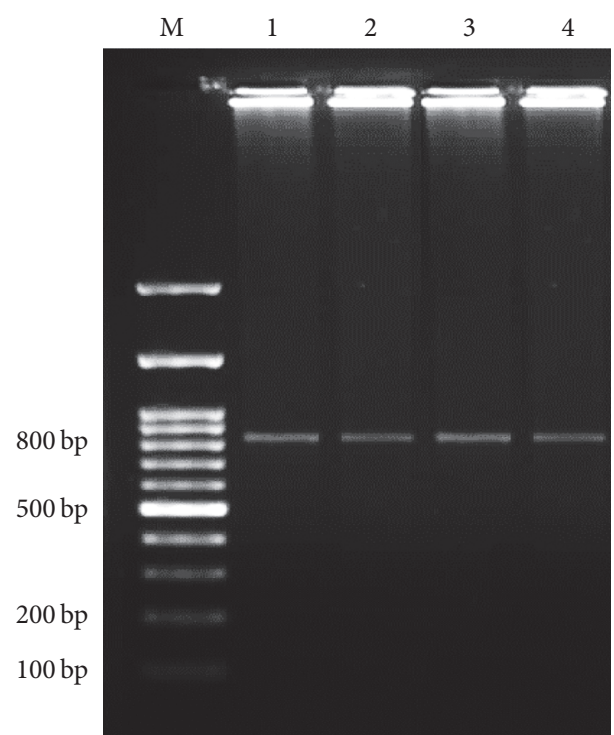

FIGURE 1: Secondary stage of nested-PCR findings on agarose gel. Lane M, DNA size marker. Lane 1, positive control for Cryptosporidium. Lanes 2-4, positive Cryptosporidium samples (830 bp).

Nucleotide sequence data reported in current article are available in the GenBank at accession numbers AB986579, $A B 986580$, and $A B 986581$.

\section{Results}

Three out of 100 samples were detected as positive for Cryptosporidium spp. using modified Ziehl-Neelsen staining under light microscope. In addition, nested-PCR was done and showed $830 \mathrm{bp}$ band which confirmed only 3 samples as Cryptosporidium spp. (Figure 1). So, overall prevalence of Cryptosporidium infection in rodents of Ahvaz city was calculated at $3 \%$ using both methods. All positive samples belonged to R. norvegicus (3/73) and from M. musculus (0/6) and $R$. rattus $(0 / 21)$ no positive cases were observed. In order to determine the genotype, PCR-RFLP technique using SspI and VspI restriction enzymes was utilized. With SspI restriction enzyme, three cuttings were seen in locations of 108 , 258 , and $421 \mathrm{bp}$ visible on agarose gel after electrophoresis. Also after using from VspI enzyme, three cuttings happened in locations 104, 106, and 600 bp (Figure 2) and indicate $C$. parvum pattern. The amplified 18s rRNA genes from PCRRFLP products of three C. parvum were sequenced. After submitting the results to the DDBJ/GenBank at accession numbers $A B 986579, \mathrm{AB} 986580$, and $A B 986581$, the nucleotide sequences were aligned with nucleotide sequences of C. parvum certified in GenBank with accession number AB986578 (Figure 3). Based on findings, C. parvum was recognized as infectious agent of Ahvaz rodents.

\section{Discussion}

Cryptosporidium spp. belong to Apicomplexa phylum with cosmopolitan distribution [1]. Rodents with maintaining the pathogens transmission cycle in surrounding regions play a key role in morbidity and mortality of human and livestock especially in areas with dense population [7].

The routine method for diagnosis of Cryptosporidium spp. is based on direct observation of oocysts in stool specimens using optical microscope. Since this method has low sensitivity and needs expertise, also it is unable to distinguish between different species of parasites; thus, molecular techniques like PCR have been used and developed recently. PCR-based techniques with high sensitivity, specificity, and rapidly features are capable of differentiating among species and genotypes in different specimens, that is, water, stool, and animal or human tissues, although they are expensive. Previously, genotyping of Cryptosporidium spp. has been done successfully by nested PCR-RFLP method according to SSU-rRNA (18S rRNA) gene [4, 18, 20, 24, 26, 27].

According to previous epidemiological reports, prevalence and rate of infection of Cryptosporidium spp. in rodents ranged from $0 \%$ to $63 \%$ and could be highly variable worldwide $[12,19]$. Present investigation is the first report which focused on the prevalence and molecular detection of Cryptosporidium spp. in wild rodents of Ahvaz city, southwest of Iran. We found 3\% (3/100) prevalence by both direct microscopic observations with Ziehl-Neelsen staining and nested-PCR (830 bp), while, in Bahrami et al. [20] survey in Tehran city (capital of Iran) using these methods, the prevalence was reported at $13 \%$ and $27.3 \%$, respectively. Also all positive samples identified C. parvum by PCR-RFLP using SspI and VspI restriction enzymes and were confirmed by sequencing that is in agreement with our study. Differences between Tehran and Ahvaz findings could be justified with sample size, location of sampling, cities population, hygienic conditions, sewage systems, and so forth. It is worth mentioning that Tehran as capital of Iran is the most crowded city with highest density population over the country which is multifold than Ahvaz. In Mashhad city (northeast of Iran), prevalence of Cryptosporidium spp. with 0\% [19] was lower than our study (3\%), while higher prevalence was reported from USA 32.8\% [13], China 11.5\% [16], Australia 8.2\% [18], Philippines $25.8 \%$ [17], and Tehran city (north of Iran) $27.3 \%$ [20].

Based on current study, 18s rRNA gene of C. parvum was detected only in $R$. norvegicus (3/73) and from $R$. rattus and $M$. musculus no positive samples were isolated. It should be mentioned that $R$. norvegicus is the most abundant rodent in southwest of Iran (73/100) and highest prevalence and rate of infection $(4.1 \%, 3 / 73)$ allocate to this species which corresponds to previous investigations $[9,11]$. The obtained results of amplifying 18s rRNA gene after sequencing were submitted to the DDBJ/GenBank under accession numbers $A B 986579, A B 986580$, and $A B 986581$ and then compared with AB986578 as control. Figure 3 illustrated several nucleotide replacements.

$\mathrm{Lv}$ and colleagues [16] studied the wild, laboratory, and pet rodents (totally 723 rodents from 18 species) in China and prevalence of Cryptosporidium spp. was reported at $11.5 \%$ and C. parvum, C. muris, C. andersoni, and C. wrairi were identified, as well. Prevalence in wild, laboratory, and pet rodents was $6.8 \%, 1.9 \%$, and $21.8 \%$, respectively. 


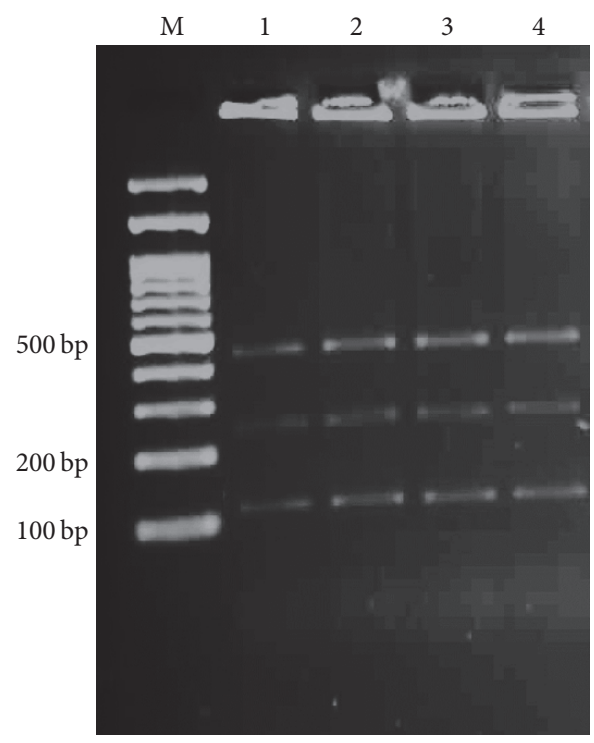

(a)

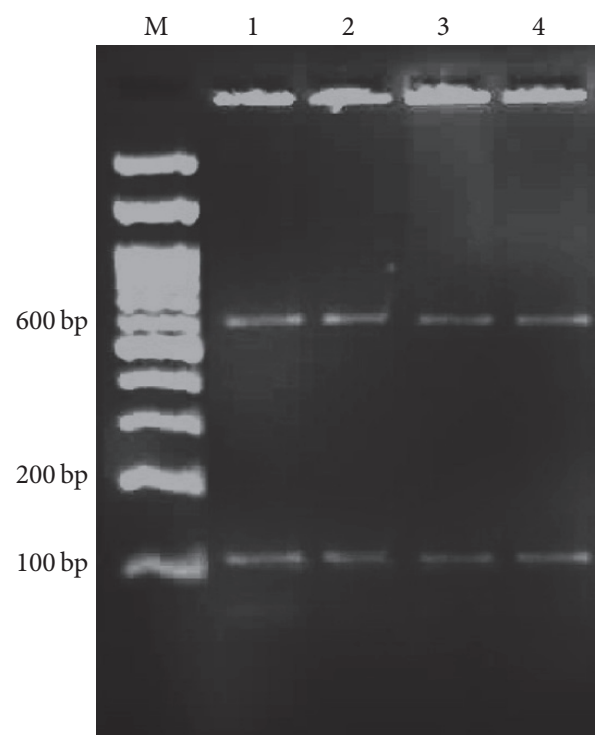

(b)

Figure 2: (a) PCR-RFLP products with SspI restriction enzyme. Three cuttings in locations 108, 258, and 421 bp are visible on gel electrophoresis. (b) PCR-RFLP products with VspI restriction enzyme. Three cuttings in locations 104, 106, and 600 bp are visible on agarose gel. Lane M, DNA size marker. Lane 1, positive control for Cryptosporidium. Lanes 2-4, positive Cryptosporidium samples.

AB986578.1 GTAATTGGGGTGGCTCATAA
AB986581. 1CCTTTCGGGTAGGTGATGGT
AB986579.1ACGGGTATAAATTGGGTTGA
AB986580.1ACCCATTTTAAATTGGGTTG

AB986578.1GTAATTGGGGTGGCTCATAA AB986581.1CCTTTCGGGTAGGTGATGGT AB986579.1ACGGGTATAAATTGGGTTGA AB986580.1ACCCATTTTAAATTGGGTTG

AB986578.1GTAATTGGGGTGGCTCATAA AB986581.1CCTTTCGGGTAGGTGATGGT AB986579.1ACGGGTATAAATTGGGTTGA AB986580.1ACCCATTTTAAATTGGGTTG

AB986578.1GTAATTGGGGTGGCTCATAA AB986581.1CCTTTCGGGTAGGTGATGGT AB986579. 1ACGGGTATAAATTGGGTTGA AB986580 . 1ACCCATTTTAAATTGGGTTG

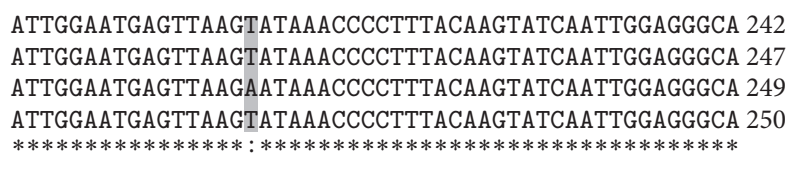

AGTCTGGTGCCAGCAGCCGCGGTAATTCCAGCTCCAATAGCGTATATTAA 292 AGTCTGGTGCCAGCAGCCGCGGTAATTCCAGCTCCAATAGCGTATATTAA 297 AGTCTGGTGCCAGCAGCCGCGGTAATTCCAGCTCCAATAGCGTATATTAA 299 AGTCTGGTGCCTGCAGCCGCGGTAATTCCAGCTCCAATAGCGTATATTAA 300 ************:****************************************

AGTTGTTGCAGTTAAAAAGCTCGTAGTTGGATTTCTGTTAATAATTTATA 342 AGTTGTTGCAGTTAAAAAGCTCGTAGTTGGATTTCTGTTAATAATTTATA 347 AGTTGTTGCAGTTAAAAAGCTCGTAGTTGGATTTCTGTTAATAATTTATA 349 AGTTGTTGCAGTTAAAAAGCTCGTAGTTGGATTTCTGTTAATAATTTATA 350

TAAATATTTTGATGAATATTTATATAATATTAACATAATTCATATTACT 392 TAAAATATTTTGATGAATATTTATATAATATTAACATAATTCATATTACT 397 TAAAATATTTTGATGAATATTTATATAATATTAACATAATTCATATTACT 399 TAAAACATTTTGAGGAATATTTATATAATATTAACATAATTCATAGTGCT 400

FIGURE 3: Multiple alignments of 18s rRNA genes from three isolates which submitted to GenBank at accession numbers $A B 986579$, $A B 986580$, and $A B 986581$. Asterisks (*) show identical nucleotides.

In another survey by $\mathrm{Ng}$-Hublin et al. [17] on 194 wild rats and mice from five species including the Asian house rat $(R$. tanezumi), the rice-field rat ( $R$. argentiventer), the Pacific rat (R. exulans), R. norvegicus, and M. musculus in Philippines, overall prevalence was reported at $25.8 \%$. In addition, based on sequencing and phylogenetic analysis of 18s rRNA gene and actin locus, C. muris, C. parvum, C. scrofarum, C. suislike genotype, and rat genotypes I-IV were recognized. In current research, only C. parvum was identified using $18 \mathrm{~s}$ rRNA gene sequencing. Throughout the world C. parvum have been isolated from numerous animals such as calves or cattle [24], some ruminants (goats and sheep) [28], horses [29], pigs [30], alpacas [31], some carnivores (gray wolves and dogs) [32, 33], reptiles [27], and rodents (rat, hamster, mice, nutria, chipmunk, squirrel, and capybara) $[13,16,17,20,34-$ 37]. In addition predominant Cryptosporidium species in Iran is C. parvum that has been verified frequently, for example, $73.3 \%$ in humans and animals [38], 83.3\% in Tehran (human samples) [39], 100\% in rodents of Tehran [20], 100\% in cattle of Ilam [24], and 68.8\% in Ahvaz (in immunocompromised patients and children) [4]. In Rafiei et al. investigation in southwest of Iran on immunocompromised patients and 
children (kidney transplant recipients, persons with hematological malignancies, HIV+ patients, and children less than 5 years old), 390 stool specimens were collected and examined. Prevalence of Cryptosporidium spp. was $4.1 \%$ (16/390). Moreover, 3 different species were identified using PCR-RFLP based on 18s rRNA gene including 11 C. parvum, 4 C. hominis, and 1 C. meleagridis [4] which was in consistent with our results. According to Rafiei et al. [4] survey and our study, C. parvum was identified as the most common species in both rodents and individuals in Ahvaz city. In past studies C. parvum transmission from rodents to human was reported repeatedly $[17,40]$.

\section{Limitations}

The present investigation was based on sampling of limited rodents species in limited areas. In future, for better understanding of exact burden of Cryptosporidium spp. and genetic diversity, studies should be designed on wide spectrum of both wild and pet rodents (rats, hamsters, mice, rabbits, etc.) in vast regions like throughout the Khuzestan province.

\section{Conclusion}

Present paper was the first report which focused on the prevalence and molecular characterization of Cryptosporidium spp. in wild rodents of Ahvaz city, southwest of Iran; that showed rate of infection in $R$. norvegicus is remarkable. Also, rodents could be potential reservoir for C. parvum. The results can help public health care to pursue new strategies (environmental sanitation, increasing the hygienic condition, increasing the hygienic condition, etc.). In future, adopting a suitable strategy for control and combat with rodents in order to decrease human cases is necessary and should be continued.

\section{Ethical Approval}

All applicable international, national, and/or institutional guidelines for the care and use of animals were followed.

\section{Competing Interests}

The authors declare that they have no conflict of interests.

\section{Authors' Contributions}

Jasem Saki contributed toward concept, design, and definition of intellectual content; Reza Asadpouri collected the samples and performed the experiments; Masoud Foroutan$\mathrm{Rad}$ contributed to the literature search and manuscript preparation; Jasem Saki and Masoud Foroutan-Rad edited and reviewed the manuscript.

\section{Acknowledgments}

This study was conducted and financially supported by Ahvaz Jundishapur University of Medical Sciences, Grant no.
CMRC-91. The authors appreciate the support of the staff of the Protozoology Laboratory at the Jundishapur University of Medical Sciences. They would like to thank Mr. Nezam Salehi for his help in collecting the samples.

\section{References}

[1] J. Plutzer and P. Karanis, "Genetic polymorphism in Cryptosporidium species: an update," Veterinary Parasitology, vol. 165, no. 3-4, pp. 187-199, 2009.

[2] M. Bouzid, P. R. Hunter, R. M. Chalmers, and K. M. Tyler, "Cryptosporidium pathogenicity and virulence," Clinical Microbiology Reviews, vol. 26, no. 1, pp. 115-134, 2013.

[3] K. Shi, F. Jian, C. Lv et al., "Prevalence, genetic characteristics, and zoonotic potential of Cryptosporidium species causing infections in farm rabbits in China," Journal of Clinical Microbiology, vol. 48, no. 9, pp. 3263-3266, 2010.

[4] A. Rafiei, Z. Rashno, A. Samarbafzadeh, and S. Khademvatan, "Molecular characterization of Cryptosporidium spp. Isolated from immunocompromised patients and children," Jundishapur Journal of Microbiology, vol. 7, no. 4, Article ID e9183, 2014.

[5] S. Gholami, M. Khanmohammadi, E. Ahmadpour et al., "Cryptosporidium infection in patients with gastroenteritis in Sari, Iran," Iranian Journal of Parasitology, vol. 9, no. 2, pp. 226-232, 2014.

[6] F. A. Nime, J. D. Burek, D. L. Page, M. A. Holscher, and J. H. Yardley, "Acute enterocolitis in a human being infected with the protozoan Cryptosporidium," Gastroenterology, vol. 70, no. 4, pp. 592-598, 1976.

[7] B. G. Meerburg, G. R. Singleton, and A. Kijlstra, "Rodent-borne diseases and their risks for public health," Critical Reviews in Microbiology, vol. 35, no. 3, pp. 221-270, 2009.

[8] R. Dehghani, H. Seyedi, S. Dehqan, and H. Sharifi, "Geographical distribution of mouse and mouse-borne diseases in Iran: a review article," Feyz, vol. 17, pp. 203-219, 2013.

[9] J. Saki and S. Khademvatan, "Detection of Toxoplasma gondii by PCR and mouse bioassay in rodents of Ahvaz District, Southwestern Iran," BioMed Research International, vol. 2014, Article ID 383859, 5 pages, 2014.

[10] Z. Seifollahi, B. Sarkari, M. H. Motazedian, Q. Asgari, M. J. Ranjbar, and S. Abdolahi Khabisi, "Protozoan parasites of rodents and their zoonotic significance in Boyer-Ahmad District, Southwestern Iran," Veterinary Medicine International, vol. 2016, Article ID 3263868, 5 pages, 2016.

[11] E. Kia, M. Homayouni, A. Farahnak, M. Mohebail, and S. Shojai, "Study of endoparasites of rodents and their zoonotic importance in Ahvaz, South West Iran," Iranian Journal of Public Health, vol. 30, pp. 49-52, 2001.

[12] J. P. Webster and D. W. Macdonald, "Cryptosporidiosis reservoir in wild brown rats (Rattus norvegicus) in the UK," Epidemiology and Infection, vol. 115, no. 1, pp. 207-209, 1995.

[13] Y. Feng, K. A. Alderisio, W. Yang et al., "Cryptosporidium genotypes in wildlife from a New York watershed," Applied and Environmental Microbiology, vol. 73, no. 20, pp. 6475-6483, 2007.

[14] M. Kváč, L. Hofmannová, S. Bertolino, L. Wauters, G. Tosi, and D. Modrý, "Natural infection with two genotypes of Cryptosporidium in red squirrels (Sciurus vulgaris) in Italy," Folia Parasitologica, vol. 55, no. 2, pp. 95-99, 2008.

[15] L. Zhou, R. Fayer, J. M. Trout, U. M. Ryan, F. W. Schaefer III, and L. Xiao, "Genotypes of Cryptosporidium species infecting 
fur-bearing mammals differ from those of species infecting humans," Applied and Environmental Microbiology, vol. 70, no. 12, pp. 7574-7577, 2004.

[16] C. Lv, L. Zhang, R. Wang et al., "Cryptosporidium spp. in wild, laboratory, and pet rodents in China: prevalence and molecular characterization," Applied and Environmental Microbiology, vol. 75, no. 24, pp. 7692-7699, 2009.

[17] J. S. Y. Ng-Hublin, G. R. Singleton, and U. Ryan, "Molecular characterization of Cryptosporidium spp. from wild rats and mice from rural communities in the Philippines," Infection, Genetics and Evolution, vol. 16, pp. 5-12, 2013.

[18] A. Paparini, B. Jackson, S. Ward, S. Young, and U. M. Ryan, "Multiple Cryptosporidium genotypes detected in wild black rats (Rattus rattus) from northern Australia," Experimental Parasitology, vol. 131, no. 4, pp. 404-412, 2012.

[19] H. Borji, J. Khoshnegah, G. Razmi, H. Amini, and M. Shariatzadeh, "A survey on intestinal parasites of golden hamster (Mesocricetus auratus) in the northeast of Iran," Journal of Parasitic Diseases, vol. 38, no. 3, pp. 265-268, 2014.

[20] F. Bahrami, J. Sadraei, and M. Frozandeh, "Molecular characterization of Cryptosporidium spp. in Wild Rats of Tehran, Iran using 18s rRNA gene and PCR_RFLP method," Jundishapur Journal of Microbiology, vol. 5, no. 3, pp. 486-490, 2012.

[21] E. Etemad, Mammals of Iran, Vol. 1 (Rodents and their identification keys), A publication of the National Society for Natural Resource and Environment Conservation, Iran, 1978.

[22] T. Fujino, T. Matsuo, M. Okada, and T. Matsui, "Detection of a small number of Cryptosporidium parvum oocysts by sugar floatation and sugar centrifugation methods," Journal of Veterinary Medical Science, vol. 68, no. 11, pp. 1191-1193, 2006.

[23] M. Pirestani, J. Sadraei, A. Dalimi Asl, M. Zavvar, and H. Vaeznia, "Molecular characterization of Cryptosporidium isolates from human and bovine using 18s rRNA gene in Shahriar county of Tehran, Iran," Parasitology Research, vol. 103, no. 2, pp. 467-472, 2008.

[24] M. Mahami Oskouei, E. Fallah, M. Ahmadi et al., "Molecular and parasitological study of Cryptosporidium isolates from cattle in Ilam, west of Iran," Iranian Journal of Parasitology, vol. 9, no. 3, pp. 435-440, 2014.

[25] M. Foroutan-Rad, S. Khademvatan, J. Saki, and M. Hashemitabar, "Holothuria leucospilota extract induces apoptosis in Leishmania major promastigotes," Iranian Journal of Parasitology, vol. 11, pp. 339-349, 2016.

[26] K. Manouchehri Naeini, M. Asadi, and M. Hashemzade Chaleshtori, "Detection and molecular characterization of Cryptosporidium species in recreational waters of Chaharmahal va Bakhtiyari province of Iran using nested-PCR-RFLP," Iranian Journal of Parasitology, vol. 6, pp. 20-27, 2011.

[27] L. Xiao, U. M. Ryan, T. K. Graczyk et al., "Genetic diversity of Cryptosporidium spp. in captive reptiles," Applied and Environmental Microbiology, vol. 70, no. 2, pp. 891-899, 2004.

[28] J. Quílez, E. Torres, R. M. Chalmers, S. J. Hadfield, E. Del Cacho, and C. Sánchez-Acedo, "Cryptosporidium genotypes and subtypes in lambs and goat kids in Spain," Applied and Environmental Microbiology, vol. 74, no. 19, pp. 6026-6031, 2008.

[29] R. M. Chalmers, A. L. Thomas, B. A. Butler, and M. C. G. Morel, "Identification of Cryptosporidium parvum genotype 2 in domestic horses," Veterinary Record, vol. 156, no. 2, pp. 4950, 2005.

[30] M. Kváč, B. Sak, D. Hanzlíková, J. Kotilová, and D. Květoňová, "Molecular characterization of Cryptosporidium isolates from pigs at slaughterhouses in South Bohemia, Czech Republic," Parasitology Research, vol. 104, no. 2, pp. 425-428, 2009.

[31] D. F. Twomey, A. M. Barlow, S. Bell et al., "Cryptosporidiosis in two alpaca (Lama pacos) holdings in the South-West of England," Veterinary Journal, vol. 175, no. 3, pp. 419-422, 2008.

[32] A. Giangaspero, R. Iorio, B. Paoletti, D. Traversa, and G. Capelli, "Molecular evidence for Cryptosporidium infection in dogs in Central Italy," Parasitology Research, vol. 99, no. 3, pp. 297-299, 2006.

[33] A. Paziewska, M. Bednarska, H. Niewegłowski, G. Karbowiak, and A. Bajer, "Distribution of Cryptosporidium and Giardia spp. in selected species of protected and game mammals from northeastern Poland," Annals of Agricultural and Environmental Medicine, vol. 14, no. 2, pp. 265-270, 2007.

[34] M. V. Meireles, R. M. Soares, F. Bonello, and S. M. Gennari, "Natural infection with zoonotic subtype of Cryptosporidium parvum in Capybara (Hydrochoerus hydrochaeris) from Brazil," Veterinary Parasitology, vol. 147, no. 1-2, pp. 166-170, 2007.

[35] A. Bajer, S. Cacciò, M. Bednarska, J. M. Behnke, N. J. Pieniazek, and E. Sinski, "Preliminary molecular characterization of Cryptosporidium parvum isolates of wildlife rodents from Poland," Journal of Parasitology, vol. 89, no. 5, pp. 1053-1055, 2003.

[36] U. Ryan, L. Xiao, C. Read, L. Zhou, A. A. Lal, and I. Pavlasek, "Identification of novel Cryptosporidium genotypes from the Czech Republic," Applied and Environmental Microbiology, vol. 69, no. 7, pp. 4302-4307, 2003.

[37] F. Berrilli, D. Di Cave, C. Orazi et al., "Giardia and Cryptosporidium in kennel dogs, pet rabbits and hamsters from Rome (Italy)," Parassitologia, vol. 48, no. 1-2, p. 261, 2006.

[38] A. R. Meamar, K. Guyot, G. Certad et al., "Molecular characterization of Cryptosporidium isolates from humans and animals in Iran," Applied and Environmental Microbiology, vol. 73, no. 3, pp. 1033-1035, 2007.

[39] A. Dalimi, F. Tahvildar-Biderouni, F. Ghaffarifar, and B. Kazemi, "The identification of human Cryptosporidium species in Tehran by PCR/RFLP," Modares Journal of Medical Sciences: Pathobiology, vol. 17, pp. 39-48, 2014.

[40] L. Xiao and R. Fayer, "Molecular characterisation of species and genotypes of Cryptosporidium and Giardia and assessment of zoonotic transmission," International Journal for Parasitology, vol. 38, no. 11, pp. 1239-1255, 2008. 


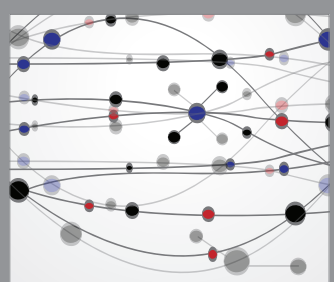

The Scientific World Journal
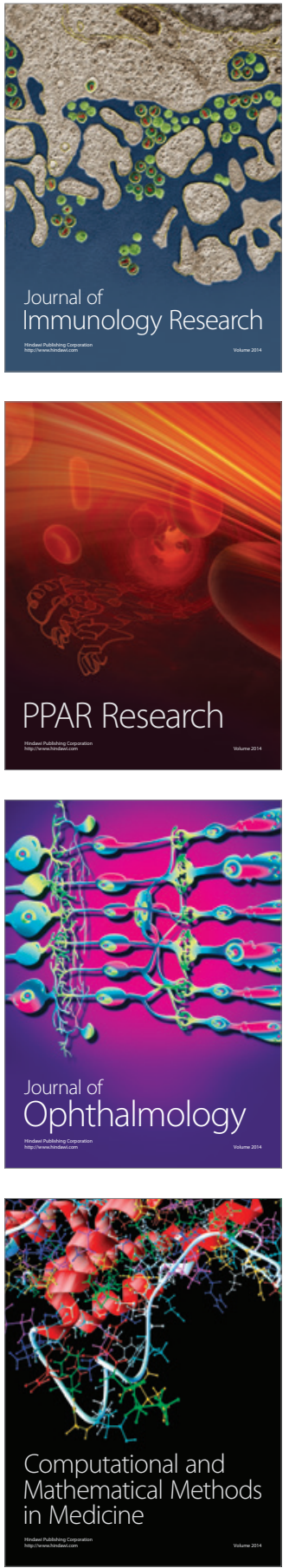

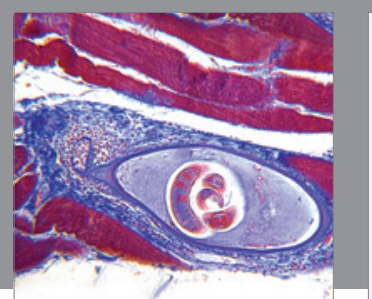

Gastroenterology Research and Practice

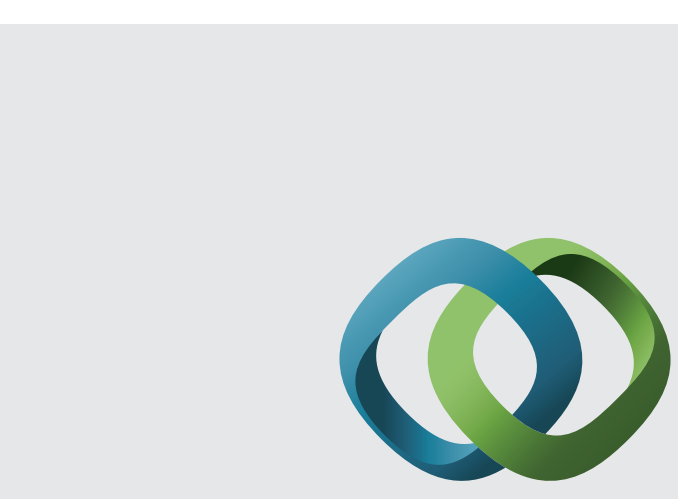

\section{Hindawi}

Submit your manuscripts at

http://www.hindawi.com
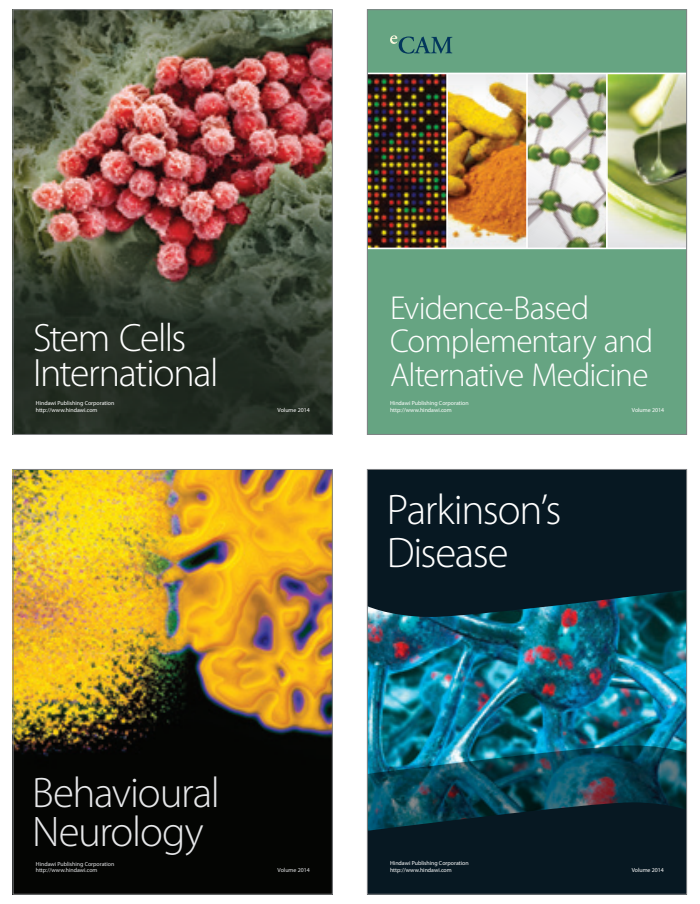
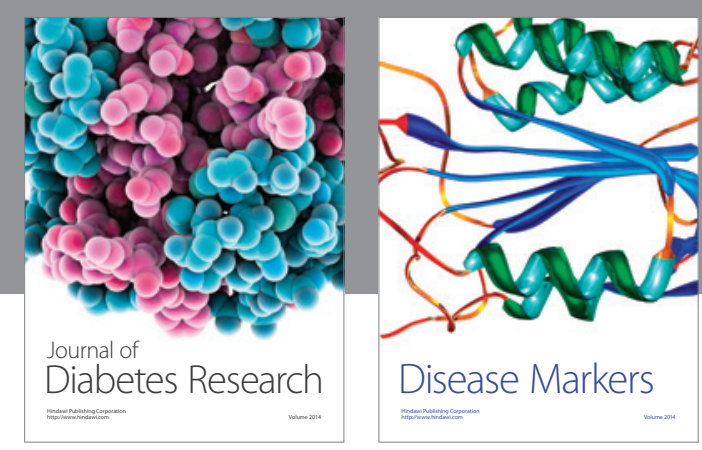

Disease Markers
